\title{
ABSOLUTE BOUNDEDNESS AND ABSOLUTE CONVERGENCE IN SEQUENCE SPACES
}

\author{
MARTIN BUNTINAS AND NAZA TANOVIĆ-MILLER
}

(Communicated by J. Marshall Ash)

\begin{abstract}
Let $\mathscr{H}$ be the set of all sequences $h=\left(h_{k}\right)_{k=1}^{\infty}$ of 0 s and $1 \mathrm{~s}$. A sequence $x$ in a topological sequence space $E$ has the property of absolute boundedness $|A B|$ if $\mathscr{H} \cdot x=\left\{y \mid y_{k}=h_{k} x_{k}, h \in \mathscr{H}\right\}$ is a bounded subset of $E$. The subspace $E_{|A B|}$ of all sequences with absolute boundedness in $E$ has a natural topology stronger than that induced by $E$. A sequence $x$ has the property of absolute sectional convergence $|A K|$ if, under this stronger topology, the net $\{h \cdot x\}$ converges to $x$, where $h$ ranges over all sequences in $\mathscr{H}$ with a finite number of $1 \mathrm{~s}$ ordered coordinatewise $\left(h^{\prime} \leq h^{\prime \prime}\right.$ iff $\forall k$, $\left.h_{k}^{\prime} \leq h_{k}^{\prime \prime}\right)$. Absolute boundedness and absolute convergence are investigated. It is shown that, for an $F K$-space $E$, we have $E=E_{|A B|}$ if and only if $E=l^{\infty} \cdot E$, and every element of $E$ has the property $|A K|$ if and only if $E=c_{0} \cdot E$. Solid hulls and largest solid subspaces of sequence spaces are also considered. The results are applied to standard sequence spaces, convergence fields of matrix methods, classical Banach spaces of Fourier series and to more recently introduced spaces of absolutely and strongly convergent Fourier series.
\end{abstract}

\section{INTRODUCTION}

We mainly use standard notation as given in $\S 2$. For an $F K$-space $E$, various forms of sectional boundedness and sectional convergence have been shown to be equivalent to invariances of the form $E=D \cdot E$ with respect to coordinatewise multiplication by some space $D$. Such statements show the equivalence of topological properties of $E$ with algebraic properties of $E$. In 1968 Garling [9] showed that an $F K$-space $E$ has the property of sectional boundedness $A B$ if and only if $E$ is invariant with respect to the space $b v$ of sequences of bounded variation, and that $E$ has the property of sectional convergence $A K$ if and only if $E=b v_{0} \cdot E$. In 1970 Buntinas [4] showed that, for an $F K$-space $E$, Cesàro sectional boundedness $\sigma B$ is equivalent to invariance with respect to the space $q$ of bounded quasiconvex sequences and that Cesàro sectional convergence $\sigma K$ is equivalent to invariance with respect to the space $q_{0}=q \cap c_{0}$ of quasiconvex null sequences. In 1973 results were obtained for more general Toeplitz sections

Received by the editors July 17, 1989 and, in revised form, January 18, 1990.

1980 Mathematics Subject Classification (1985 Revision). Primary 46A45; Secondary 42A16, 42A28.

Research partially supported by U.S.-Yugoslav Joint Fund (NSF JF 803). 
[5]. In 1977, Sember [12] and Sember and Raphael [13] showed that for $F K$ spaces, unrestricted sectional boundedness $U A B$ is equivalent to invariance with respect to the space $c$ of convergent sequences and that unrestricted sectional convergence $U A K$ is equivalent to invariance with respect to the space $c_{0}$ of null sequences.

In this paper we study absolute boundedness $|A B|$ and absolute convergence $|A K|$. These conditions are stronger than $U A B$ and $U A K$, respectively. However, for $F K$-spaces, we show that the property $|A K|$ is equivalent to $U A K$. Among other results, we show in $\S 3$ that an $F K$-space $E$ has absolute boundedness if and only if it is solid ( $l^{\infty}$-invariant) and that it has absolute convergence if and only if it is $c_{0}$-invariant.

The intersection of all solid $F K$ containing an $F K$-space $E$ is called the solid hull of $E$. In $\S 4$ we show that it is an $F K$-space and characterize it as an $F K$-product space. We show that the solid hull of an $F K$-space $E$ is related by duality to the $|A B|$ subspace of $E$.

In the last section, we give examples and applications to summability theory and Fourier analysis.

\section{Definitions}

Let $\omega$ be the space of all real or complex sequences $x=\left(x_{k}\right)$. An $F K$ space is a subspace of $\omega$ with a complete metrizable locally convex topology with continuous coordinate functionals $f_{k}: x \rightarrow x_{k}$ for all $k$. An $F K$-space whose topology is defined by a norm is a Banach space and is called a $B K$ space. Let $e^{k}$ be the sequence with 1 in the $k$ th coordinate and 0 elsewhere, and let $\varphi$ be the linear span of $\left\{e^{1}, e^{2}, e^{3}, \ldots\right\}$. In this paper we consider only $F K$ - and $B K$-spaces containing $\varphi$, although all the definitions apply to more general $K$-spaces containing $\varphi$.

We use the notation $x \cdot y:=\left(x_{k} y_{k}\right)$ for the coordinatewise product of sequences $x$ and $y$ and, for subsets $A$ and $B$ of $\omega$, we use $A \cdot B:=\{x \cdot y \mid x \in$ $A, y \in B\}$. If $D \subset \omega$ and $F$ is an $F K$-space, we define the $F$-dual of $D$ as the multiplier space $D^{F}=(D \rightarrow F):=\{y \in \omega \mid x \cdot y \in F$ for all $x \in D\}$. Let $s^{n}:=\sum_{k=1}^{n} e^{k}=(1,1, \ldots, 1,0, \ldots)$ and let $e:=(1,1,1, \ldots)$ be the sequence of all "ones." The $n$th section of a sequence $x$ is $s^{n} x:=s^{n} \cdot x=$ $\left(x_{1}, x_{2}, \ldots, x_{n}, 0, \ldots\right)$.

A sequence $x$ in $\omega$ has the property $A B$ of sectional boundedness in an $F K$-space $E$ if the sections $s^{n} x$ of $x$ form a bounded subset of $E$, and it has the property $A K$ of sectional convergence if, in addition, the sections converge to $x$ in the topology of $E$.

Let $\mathscr{H}:=\left\{h \in \omega \mid h_{k}=1\right.$ or $h_{k}=0$ for all $\left.k\right\}$ and $\mathscr{H}_{\varphi}:=\mathscr{H} \cap \varphi$. The unconditional (or unrestricted) sections of a sequence $x$ are the sequences in the set $\mathscr{H}_{\varphi} \cdot x$. The absolute set of $x$ is $\mathscr{H} \cdot x$. Since $e \in \mathscr{H}$, we have $x \in \mathscr{H} \cdot x$. Let $E$ be an $F K$-space and let $x \in \omega$. We say that $x$ has the property $U A B$ of unconditional sectional boundedness in $E$ if $\mathscr{H}_{\varphi} \cdot x$ is a bounded subset of 
$E$ and we say that $x$ has the property $|A B|$ of absolute boundedness if $\mathscr{H} \cdot x$ is a bounded subset of $E$.

For each $F K$-space $E$, we define the space $E_{A B}$ consisting of all elements $x$ of $\omega$ with the property $A B$ in $E$. Similarly, for the properties $U A B$ and $|A B|$, we obtain spaces $E_{U A B}$ and $E_{|A B|}$. That is,

$$
\begin{aligned}
& E_{A B}=\left\{x \in \omega \mid\left\{s^{n} x\right\}_{n=1}^{\infty} \text { is a bounded subset of } E\right\}, \\
& E_{U A B}=\left\{x \in \omega \mid \mathscr{H}_{\varphi} \cdot x \text { is a bounded subset of } E\right\}, \\
& E_{|A B|}=\{x \in \omega \mid \mathscr{H} \cdot x \text { is a bounded subset of } E\} .
\end{aligned}
$$

Each of these spaces is an $F K$-space under an appropriate topology discussed in $\S 3$. These spaces are not necessarily subspaces of $E$, as is shown by the example $\left(c_{0}\right)_{U A B}=\left(c_{0}\right)_{A B}=l^{\infty}$. However, $E_{|A B|}$ is always a subspace of $E$, since $e \in \mathscr{H}$. We say that an $F K$-space $E$ has the property $A B, U A B$, or $|A B|$ if $E$ is a subset of $E_{A B}, E_{U A B}$, or $E_{|A B|}$, respectively. Clearly we have $E_{|A B|} \subset E_{U A B} \subset E_{A B}$. The converse inclusions do not generally hold. For example, the $B K$-space $c$ of all convergent sequences has the property $U A B$ but it does not have the property $|A B|$.

The set $\mathscr{H}_{\varphi}$ is a directed set under the relation $h^{\prime \prime} \geq h^{\prime}$ defined by $h_{k}^{\prime \prime} \geq h_{k}^{\prime}$ for all $k$. A sequence $x$ in an $F K$-space $E$ containing $\varphi$ has the property $U A K$ in $E$ if the net $h \cdot x$, where $h$ ranges over $\mathscr{H}_{\varphi}$, converges to $x$ under the topology of $E$. We say that $x$ has the property $|A K|$ of absolute sectional convergence if $\mathscr{H} \cdot x \subset E$ and the net $h \cdot h^{\prime} \cdot x$, where $h$ ranges over $\mathscr{H}_{\varphi}$, converges to $h^{\prime} \cdot x$ uniformly in $h^{\prime} \in \mathscr{H}$ under the topology of $E$.

We define $E_{A K}$ to be the space of all elements $x$ of $E$ with the property $A K$ in $E$. The same can be done for the properties $U A K$ and $|A K|$. That is,

$$
\begin{aligned}
& E_{A K}=\left\{x \in E \mid \lim _{n} s^{n} x=x\right\}, \\
& E_{U A K}=\left\{x \in E \mid \lim _{h} h \cdot x=x, h \in \mathscr{H}_{\varphi}\right\}, \\
& E_{|A K|}=\left\{x \in E \mid \lim _{h} h \cdot h^{\prime} \cdot x=h^{\prime} \cdot x, \text { uniformly in } h^{\prime} \in \mathscr{H},\right. \text { for } \\
& \left.h \in \mathscr{H}_{\varphi}\right\} .
\end{aligned}
$$

The space $E_{A D}$ is the closure of $\varphi$ in $E$. Since $\varphi \subset E$, we have the inclusions $\varphi \subset E_{|A K|} \subset E_{U A K} \subset E_{A K} \subset E_{A D} \subset E$. If $E_{A D}=E$, we say that $E$ has the property of sectional density $A D$. If $y \in E$ whenever $\left|y_{k}\right| \leq\left|x_{k}\right|$ for some $x \in E$, we say that $E$ is solid; this is equivalent to $l^{\infty}$-invariance: $E=l^{\infty} \cdot E$.

We finish this section with a list of some $B K$-spaces and their norms. The $B K$-spaces $l^{\infty}, c$, and $c_{0}$ are the space of all bounded, convergent, and null sequences $x$, respectively, under the sup norm $\|x\|_{\infty}:=\sup _{k}\left|x_{k}\right| ; b v$ is the $B K$-space of all sequences $x$ of bounded variation under the norm $\|x\|_{b v}:=$ $\sum_{k=1}^{\infty}\left|x_{k}-x_{k+1}\right|+\|x\|_{\infty} ; b v_{0}=b v \cap c_{0}$ under the same norm; $c s$ is the $B K$-space of sequences $x$ with convergent series under the norm $\|x\|_{b s}:=$ $\sup _{n}\left|\sum_{k=1}^{n} x_{k}\right| ; l^{p}$, for $1 \leq p<\infty$, are the $B K$-spaces of sequences $x$ with absolutely $p$-summable series under the norm $\|x\|_{p}:=\left(\sum_{k=1}^{\infty}\left|x_{k}\right|^{p}\right)^{1 / p}$; the mixed $l^{p, q}$ spaces $(1 \leq p \leq \infty, 1 \leq q<\infty)$ [11] consist of all $x$ with 
$\|x\|_{p, q}:=\left(\sum_{j=1}^{\infty}\left(\left\|d^{j} x\right\|_{p}\right)^{p}\right)^{1 / q}<\infty$ where $d^{j} x_{k}=x_{k}$ for $2^{j} \leq k<2^{j+1}$ and $d^{j} x_{k}=0$ elsewhere; for $q=\infty,\|x\|_{p, q}:=\sup _{j}\left\|d^{j} x\right\|_{p}$. Clearly $l^{p, p}=l^{p}$.

\section{AbSOLUTE BoundedNESS AND ABSOLUTE CONVERGENCE}

The properties $U A B$ and $U A K$ were investigated by Sember [12] and Sember and Raphael [13]. The properties $|A B|$ and $|A K|$ considered here are related. Let $E$ be a $B K$-space under the norm $\|x\|_{E}$. We define the (extended) absolute norm on $E$ by

$$
\|x\|_{|E|}:=\sup _{h \in \mathscr{C}}\|h \cdot x\|_{E},
$$

with the convention that $\|h \cdot x\|_{E}=\infty$ whenever $h \cdot x \notin E$. Clearly, $\|x\|_{E} \leq$ $\|x\|_{|E|}$ and $E_{|A B|}=\left\{x \in E \mid\|x\|_{|E|}<\infty\right\}$.

Similary, if $E$ be an $F K$-space with an increasing family of seminorms $p^{1} \leq p^{2} \leq p^{3} \leq \cdots$ defining the topology of $E$, we define the (extended) absolute seminorms by

$$
p_{|E|}^{k}(x):=\sup _{h \in \mathscr{E}} p^{k}(h \cdot x), \quad k=1,2,3, \ldots,
$$

with the convention that $p^{k}(h \cdot x)=\infty$ whenever $h \cdot x \notin E$. Clearly, $E_{|A B|}=$ $\left\{x \in E \mid p_{|E|}^{k}(x)<\infty\right.$ for $\left.k=1,2, \ldots\right\}$. By Garling's Theorem [9, p. 998], $E_{|A B|}$ is an $F K$-space under the seminorms $p^{1}, p_{|E|}^{1}, p^{2}, p_{|E|}^{2}, \ldots$. Since $p^{k} \leq$ $p_{|E|}^{k}$ for all $k, p^{1}, p^{2}, p^{3}, \ldots$ may be omitted. Hence the following theorem and corollary hold:

Theorem 1. Let $E$ be an $F K$-space with defining seminorms $p^{1}, p^{2}, p^{3}, \ldots$ Then $E_{|A B|}$ is an $F K$-space whose topology is defined by the seminorms $p_{|E|}^{1}$, $p_{|E|}^{2}, p_{|E|}^{3}, \ldots$.

Corollary. If $E$ is a BK-space under the norm $\|\cdot\|_{E}$, then $E_{|A B|}$ is a BK-space under the norm $\|\cdot\|_{|E|}$.

Remark. It follows that a sequence $x$ in an $F K$-space $E$ has the property $|A K|$ if and only if the net $\mathscr{H}_{\varphi} \cdot x$ converges to $x$ in the topology of $E_{|A B|}$.

Theorem 2. Let $E$ be an $F K$-space containing $\varphi$. Then the following statements are equivalent:

[a] $x \in E_{|A B|}$;

[b] $\mathscr{H} \cdot x \subset E$; and

[c] $l^{\infty} \cdot x \subset E$.

Proof. We have $[a] \Rightarrow[b]$ by definition of $|A B|$. Suppose $[b]$. Then $\mathscr{H} \subset$ $(\{x\} \rightarrow E)$. The multiplier space $(\{x\} \rightarrow E)$ is an $F K$-space [10, p. 229]. By Bennett and Kalton [3], $\mathscr{H}$ is a subset of an $F K$-space if and only if $l^{\infty}$ is a subset. Thus $l^{\infty} \subset(\{x\} \rightarrow E)$, or $l^{\infty} \cdot x \subset E$. Thus $[b] \Rightarrow[c]$. Finally suppose 
[c]. Let $T_{x}$ be the multiplier map from $l^{\infty}$ to $E$ defined by $T_{x}(y)=x \cdot y$. By the Closed Graph Theorem, all multiplier maps between $F K$-spaces are continuous [20]. Since $\mathscr{H}$ is a bounded subset of $l^{\infty}, T_{x}(\mathscr{H})=x \cdot \mathscr{H}$ is a bounded subset of $E$. Thus $x$ has the property $|A B|$ in $E$.

Since $e \in l^{\infty}$, we have $\left(l^{\infty} \rightarrow E\right) \subset E$. Anderson and Shields [1] have observed that $\left(l^{\infty} \rightarrow E\right)$ is the largest solid subspace of $E$. By $[c]$ above, we obtain the following.

Corollary 1. Let $E$ be an $F K$-space containing $\varphi$. Then $E_{|A B|}=\left(l^{\infty} \rightarrow E\right)$. This is the largest solid subspace of $E$.

Corollary 2. An $F K$-space has the property $|A B|$ if and only if it is solid.

Corollary 3. For any $F K$-space $E, E_{U A B}$ is solid.

Proof. If $E$ is an $F K$-space under the seminorms $p^{k}, k=1,2,3, \ldots$, then $E_{U A B}$ is an $F K$-space under the seminorms $\sup _{h \in \mathscr{H}_{\varphi}} p^{k}(h \cdot x)$. Since $\mathscr{H}_{\varphi}=$ $\mathscr{H}_{\varphi} \cdot \mathscr{H}_{\varphi}=\mathscr{H}_{\varphi} \cdot \mathscr{H}$, it follows that $\mathscr{H}_{\varphi} \cdot x$ is a bounded subset of $E$ if and only if $\mathscr{H}_{\varphi} \cdot x$ is a bounded subset of $E_{U A B}$. This is true if and only if $\mathscr{H} \cdot x$ is a bounded subset of $E_{U A B}$. That is, $E_{U A B}=\left(E_{U A B}\right)_{U A B}=\left(E_{U A B}\right)_{|A B|}$, which is solid.

The space $E_{U A B}$ can be characterized as follows.

Theorem 3. Let $E$ be an $F K$-space containing $\varphi$. Then $E_{U A B}=\left(c_{0} \rightarrow E\right)$. Proof. By [12, Theorem 4] $c_{0} \cdot E_{U A B} \subset E$. Thus $E_{U A B} \subset\left(c_{0} \rightarrow E\right)$. Conversely, suppose $c_{0} \cdot x \subset E$. Define the map $T_{x}: c_{0} \rightarrow E$ by $T_{x}(y)=x \cdot y$. Since $T_{x}$ is continuous, $T_{x}$ takes bounded subsets of $c_{0}$ into bounded subsets of $E$. Let $U$ be the unit sphere of $c_{0}$. Then $U \cdot x$ is bounded in $E$. By [12, Theorem $3]$, we have $x \in E_{U A B}$.

Corollary. Let $E$ be an $F K$-space containing $\varphi$. Then $E \cap E_{U A B}=(c \rightarrow E)$.

In the same way, we can use the results in [9] to show that

$$
E_{A B}=\left(b v_{0} \rightarrow E\right) \text { and } E \cap E_{A B}=(b v \rightarrow E) .
$$

Although $E_{U A B}$ is solid, $E_{U A B} \cap E$ need not be solid. This is the case when $E=c$. Thus, $E_{|A B|}$ is generally a proper subspace of $E_{U A B} \cap E$. Also, if $E \subset E_{U A B}$, the space $E_{U A B}$ need not be the smallest solid space containing $E$. The space $\left(c_{0}\right)_{U A B}=l^{\infty}$ provides an example.

Theorem 4. Let $E$ be an $F K$-space. If $E_{U A B} \subset E$, then $E_{|A B|}=E_{U A B}$. Proof. Clearly, $E_{|A B|} \subset E_{U A B}$. By Theorem 2, Corollary 3, $E_{U A B}$ is solid. Since $E_{|A B|}$ is the largest solid subspace of $E$, the statement follows.

Theorem 5. Let $E$ be an $F K$-space containing $\varphi$. Then the following statements are equivalent:

[a] $E$ is solid and has the property $A D$; 
[b] $E$ is solid and has the property $A K$;

[c] $E=c_{0} \cdot E$;

[d] $E$ has the property $U A K$; and

[e] $E$ has the property $|A K|$.

Proof. The equivalence of $[a],[b]$, and $[c]$ was proved by Garling [9, p. 1007]. $[b] \Rightarrow[e]$ : Fremlin and Garling $[9$, p. 1006] showed that a solid $F K$-space $E$ is locally solid; that is, the topology of $E$ is defined by seminorms $p$ with the property $p(d \cdot x) \leq p(x)$ for all sequences $d$ in the unit sphere of $l^{\infty}$. Let $p$ be such a continuous seminorm, let $x \in E$, and let $\varepsilon>0$. Then $p_{|E|}=p$. Suppose $p\left(s^{n} x-x\right)<\frac{\varepsilon}{2}$, and let $h \in \mathscr{H}$ such that $h \geq s^{n}$. Then $s^{n}=h \cdot s^{n}$. Hence $p_{|E|}(h \cdot x-x) \leq p_{|E|}\left(h \cdot x-s^{n} x\right)+p_{|E|}\left(s^{n} x-x\right)=p_{|E|}\left(h \cdot x-h \cdot s^{n} x\right)+p_{|E|}\left(s^{n} x-x\right)=$ $p\left(h \cdot\left(x-s^{n} x\right)\right)+p\left(s^{n} x-x\right) \leq 2 p\left(s^{n} x-x\right)<\varepsilon$. This shows that the net $\mathscr{H} \cdot x$ converges to $x$ under the topology of $E_{|A B|} \cdot[e] \Rightarrow[d]$ is immediate from the definitions. $[d] \Rightarrow[b]$ : The property $U A K$ clearly implies $A K$, since $s^{n} \in \mathscr{H}_{\varphi}$ for all $n$. Sember and Raphael [13, Corollary 3.2] have shown that $E_{U A K}$ is solid. Since $E=E_{U A K}$, it follows that $E$ is solid.

Remark. A $B K$-space has the property $|A K|$ if and only if, for all $x \in E$, $\left\|s^{n} x-x\right\|_{|E|} \rightarrow 0$ as $n \rightarrow \infty$. Moreover, in this case $\|x\|_{|E|}=\sup \left\|s^{n} x\right\|_{|E|}$. A similar statement can be made about $F K$-spaces.

Theorem 6. If $E$ is an $F K$-space containing $\varphi$ and $E_{A D}$ is solid, then $E_{A D}=$ $E_{A K}=E_{U A K}=E_{|A K|}=c_{0} \cdot E$.

Proof. Clearly $E_{|A K|} \subset E_{U A K} \subset E_{A K} \subset E_{A D}$. Since $E_{A D}$ is a closed subspace of $E$, it is an $F K$-space under the subspace topology. Hence $E_{|A K|}=\left(E_{A D}\right)_{|A K|}$. By Theorem $5([a] \Rightarrow[e])$, we have $\left(E_{A D}\right)_{|A K|}=E_{A D}$.

Corollary 1. If $E$ is an $F K$-space containing $\varphi$ with the property $U A B$, then $E_{A D}=E_{A K}=E_{U A K}=E_{|A K|}=c_{0} \cdot E$.

Proof. If $E$ has the property $U A B$, then by Sember and Raphael [13, Theorem 4], $E_{A D}=E_{U A K}=c_{0} \cdot E$. Thus $E_{A D}$ is solid and satisfies the conditions of Theorem 6.

If $E$ is solid, then $E$ has the property $U A B$ since $E=E_{|A B|} \subset E_{U A B}$.

Corollary 2. If $E$ is a solid $F K$-space containing $\varphi$, then $E_{A D}=E_{A K}=E_{U A K}=$ $E_{|A K|}=c_{0} \cdot E$.

Corollary 3. For any $F K$-space $E$ containing $\varphi, E_{|A K|}=c_{0} \cdot E_{|A B|}$.

Proof. By definition, $E_{|A K|}=\left(E_{|A B|}\right)_{U A K}$. Since $E_{|A B|}$ is solid, we have

$$
\left(E_{|A B|}\right)_{U A K}=c_{0} \cdot E_{|A B|}
$$

by Corollary 2 .

Theorem 7. Let $E$ be an $F K$-space. Then $E_{U A K}=E_{|A K|}$. 
Proof. Let $x \in E$. The statement $x \in E_{U A K}$ means that the net $\mathscr{H}_{\varphi} \cdot x$ converges to $x$ in the topology of $E$. The statement $x \in E_{|A K|}$ means that the net $\mathscr{H}_{\varphi} \cdot x$ converges to $x$ in the topology of $E_{|A B|}$; that is, $E_{|A K|}=\left(E_{|A B|}\right)_{U A K}$. Also $E_{U A K}=c_{0} \cdot E_{U A B}=c_{0} \cdot\left(E_{U A B}\right)_{U A B}=\left(E_{U A B}\right)_{U A K}$. It remains to be shown that $\left(E_{|A B|}\right)_{U A K}=\left(E_{U A B}\right)_{U A K}$, which by Theorem 6, Corollary 2 is equivalent to $\left(E_{|A B|}\right)_{A K}=\left(E_{U A B}\right)_{A K}$. Since $E_{|A B|} \subset E_{U A B}$, we have the inclusion $\left(E_{|A B|}\right)_{A K} \subset\left(E_{U A B}\right)_{A K}$. Conversely, suppose $x \in\left(E_{U A B}\right)_{A K}$. Then, for each continuous seminorm $p$ on $E, \sup _{h \in \mathscr{E}_{\phi}} p\left(h \cdot\left(s^{n} x-s^{m} x\right)\right) \rightarrow 0$ as $n, m \rightarrow \infty$. Since $s^{n} x-s^{m} x \in \varphi$, we have $\sup _{h \in \mathscr{E}} p\left(h \cdot\left(s^{n} x-s^{m} x\right)\right) \rightarrow 0$ as $n, m \rightarrow \infty$; that is, $x \in\left(E_{|A B|}\right)_{A K}$.

\section{THE SOLID HULL OF AN $F K$-SPACE}

For an $F K$-space $E$, the solid hull $\langle E\rangle$ is the intersection of all solid $F K$ spaces containing $E$. It is clearly solid. The solid hull was investigated by Anderson and Shields in [1]. We show that the solid hull is an $F K$-product space, and we find a dual relationship between $E_{|A B|}$ and $\langle E\rangle$.

The $F K$-product $E \hat{\otimes} F$ of two $F K$-spaces $E$ and $F$ was defined in [6] and [7] and was characterized as the smallest $F K$-space containing the coordinate product $E \cdot F$. If $E$ and $F$ are $B K$-spaces, then $E \hat{\otimes} F$ turns out to be a $B K$-space.

Theorem 8. Let $E$ be an FK-space. The solid hull of $E$ is the $F K$-space $l^{\infty} \hat{\otimes} E$.

Proof. If $F$ is a solid $F K$-space containing $E$, then $F=l^{\infty} \cdot F \supset l^{\infty} \cdot E \supset$ $E$. Thus $F \supset l^{\infty} \hat{\otimes} E \supset E$. But $l^{\infty} \hat{\otimes} E$ is itself solid, since $l^{\infty} \hat{\otimes}\left(l^{\infty} \hat{\otimes} E\right)=$ $\left(l^{\infty} \hat{\otimes} l^{\infty}\right) \hat{\otimes} E=l^{\infty} \hat{\otimes} E$. Thus it is the smallest solid $F K$-space containing $E$.

[7, Theorem 4.3] states that $(E \hat{\otimes} F)_{A D}=(E \hat{\otimes} F)_{A K}=E_{A K} \hat{\otimes} F$ whenever $E \subset E_{A B}$. We obtain the following:

Corollary. Let $E$ be an $F K$-space containing $\varphi$. Then $\left(l^{\infty} \hat{\otimes} E\right)_{A D}=\left(l^{\infty} \hat{\otimes} E\right)_{A K}$ $=c_{0} \hat{\otimes} E$.

From this corollary we see that if $E$ is solid and has the property $A D$, then $E=c_{0} \cdot E$. This is Theorem $5([a] \Rightarrow[c])$. Other parts of Theorems 5 and 6 can also be obtained.

The next theorem exhibits a dual relationship between the space $E_{|A B|}$ and the solid hull $\langle E\rangle$.

Theorem 9. Let $E$ and $F$ be $F K$-spaces. Then $(\langle E\rangle \rightarrow F)=(E \rightarrow F)_{|A B|}$. That is, the F-dual of the solid hull of $E$ is the largest solid subspace of the $F$-dual of $E$.

Proof. By Theorem 2, Corollary 1, $(E \rightarrow F)_{|A B|}=\left(l^{\infty} \rightarrow(E \rightarrow F)\right)$. By [7, (5.6)], $\left(l^{\infty} \rightarrow(E \rightarrow F)\right)=\left(\left(l^{\infty} \hat{\otimes} E\right) \rightarrow F\right)$. 
For example, let the $\alpha$ - and $\beta$-duals of $E$ be defined by $E^{\alpha}=\left(E \rightarrow l^{1}\right)$ and $E^{\beta}=(E \rightarrow c s)$, respectively. We have $E^{\alpha}=\left(E^{\alpha}\right)_{|A B|}=\langle E\rangle^{\alpha}$. Also $\left(E^{\beta}\right)_{|A B|}=\left(l^{\infty} \rightarrow E^{\beta}\right)=\left(E^{\beta \beta} \rightarrow l^{1}\right)=E^{\beta \beta \alpha}=E^{\alpha}$ [7, Theorem (5.1)]; [8, Theorem 1]. Similarly $(\langle E\rangle)^{\beta}=E^{\alpha}$.

Corollary. For any $F K$-space $E$, we have $E^{\alpha \alpha}=\langle E\rangle_{A B}=\langle E\rangle_{U A B}$.

Proof. By Theorem 9, we have $\langle E\rangle^{\alpha}=\left(E^{\alpha}\right)_{|A B|}$. Also, $\left(E^{\alpha}\right)_{|A B|}=E^{\alpha}$, since $E^{\alpha}$ is solid. Thus $\langle E\rangle^{\alpha \alpha}=E^{\alpha \alpha}$. But $\langle E\rangle^{\alpha \alpha}=\langle E\rangle_{A B}$ by [5, Theorem 4] and [8, Remark (6)]. As noted after Theorem 3, $\langle E\rangle_{A B}=\left(b v_{0} \rightarrow\langle E\rangle\right)$. This is a subset of $\left(c_{0} \rightarrow\langle E\rangle\right)$, which is $E_{U A B}$ by Theorem 3. That is, $\langle E\rangle_{A B} \subset\langle E\rangle_{U A B}$, and thus $\langle E\rangle_{A B}=\langle E\rangle_{U A B}$.

\section{EXAMPLES AND APPLICATIONS}

The properties $|A B|$ and $|A K|$ are strong properties of $F K$-spaces. We have the following list:

$$
\begin{aligned}
& l_{|A K|}^{\infty}=c_{0}, \quad l_{|A B|}^{\infty}=\left\langle l^{\infty}\right\rangle=l^{\infty} ; \\
& c_{|A K|}=c_{|A B|}=c_{0}, \quad\langle c\rangle=l^{\infty} ; \\
& c s_{|A K|}=c s_{|A B|}=l^{1}, \quad\langle c s\rangle=c_{0} ; \\
& b v_{|A K|}=b v_{|A B|}=l^{1}, \quad\langle b v\rangle=l^{\infty} ; \\
& l_{|A K|}^{p}=l_{|A B|}^{p}=\left\langle l^{p}\right\rangle=l^{p}(1 \leq p<\infty) ; \text { and } \\
& l_{|A K|}^{p, q}=l_{|A B|}^{p, q}=\left\langle l^{p, q}\right\rangle=l^{p, q}(1 \leq p<\infty, 1 \leq q \leq \infty) .
\end{aligned}
$$

Given an infinite matrix $T=\left(t_{n k}\right)$ of real or complex numbers, let $c_{T}$ denote the convergence field of $T$; i.e., $c_{T}=\{x \in \omega: T x \in c\}$. By the above list we have $c s_{|A B|}=l^{1}$. We will now show that this can be extended to convergence fields $c_{T}$ of all series-sequence regular matrices $T$ (i.e., $c_{T} \supset c s$ and $\lim _{n} \sum_{k} t_{n k} x_{k}=\sum_{k} x_{k}$ for all $x \in c s$ ).

Theorem 10. If $T$ is a matrix with $\lim _{n \rightarrow \infty} t_{n k}=1$ for each $k$, then $\left(c_{T}\right)_{|A B|} \subset$ $l^{1}$.

Proof. The space $c_{T}$ is an $F K$-space under the seminorms $p^{k}(x)=\left|x_{k}\right|(k=$ $1,2,3, \ldots), q^{n}(x)=\sup _{m}\left|\sum_{k=1}^{m} t_{n k} x_{k}\right|(n=1,2,3, \ldots)$ and $r(x)=$ $\sup _{n}\left|\sum_{k} t_{n k} x_{k}\right|,[20],[10]$. By Theorem $1,\left(c_{T}\right)_{|A B|}$ is an $F K$-space under the seminorms $p_{\left|c_{T}\right|}, q_{\left|c_{T}\right|}^{n}$, and $r_{\left|c_{T}\right|}$. Let $\|x\|=\sup _{n} \sum_{k}\left|t_{n k} x_{k}\right|$. It can be easily verified that $\left(c_{T}\right)_{|A B|}$ is a $B K$-space under the norm $\|\cdot\|$ since $2 r_{\left|c_{T}\right|} \geq\|\cdot\| \geq r_{\left|c_{T}\right|}=\sup _{n} q_{\left|c_{T}\right|}^{n} \geq \sup _{k} p_{\left|c_{T}\right|}^{k}$. Furthermore $\|x\| \geq \sum_{k}\left|x_{k}\right|$ for all $x \in\left(c_{T}\right)_{|A B|}$.

Every series-sequence regular matrix $T$ satisfies the conditions of Theorem 10. This can be shown by considering the sequences $e^{k}, k=1,2,3, \ldots$. 
Corollary. If $T$ is a series-sequence regular matrix, then $\left(c_{T}\right)_{|A B|}=l^{1}$.

We now apply the concepts considered in this paper to the spaces of Fourier coefficients of some classes of functions. Let $L^{p}(p \geq 1)$ be the Banach space of all real- or complex-valued $2 \pi$-periodic integrable functions with the norm $\|f\|_{L^{p}}=\left(\frac{1}{2 \pi} \int|f|^{p}\right)^{1 / p}$, where the integral is taken over any interval of length $2 \pi$. Let $C$ be the Banach space of all continuous real- or complex-valued $2 \pi$-periodic functions with the norm $\|f\|_{C}=\sup _{x}|f(x)|$.

If $f \in L^{1}$, let $\hat{f}(k), k \in \mathbb{Z}$, denote the $k$ th complex Fourier coefficient of $f, \hat{f}=(\hat{f}(k))_{k \in \mathbb{Z}}$ and let $s_{n} f, n=0,1, \ldots$ denote the $n$th partial sum of the Fourier series of $f$. If $E$ is a subspace of $L^{1}$, let $\widehat{E}$ denote the class of all sequences of Fourier coefficients of functions in $E$, i.e., $\widehat{E}=\{\hat{f}: f \in E\}$. Although the results in the preceding sections are for spaces of one-way sequences, they can be easily extended to the classes $\widehat{E}$ of two-way sequences. If $E$ is a Banach space, then $\widehat{E}$ is a Banach space under the induced norm $\|\hat{f}\|_{\widehat{E}}:=\|f\|_{E}$, and conversely. Given a Banach space $E$ contained in $L^{1}$ we can determine the corresponding subspaces of absolutely bounded and absolutely convergent Fourier series, in the topology of $E$, by determining the spaces $\widehat{E}_{|A B|}$ and $\widehat{E}_{|A K|}$. We shall also consider the corresponding solid hull $\langle\widehat{E}\rangle$.

Two classical spaces of functions in Fourier analysis, determined by the pointwise convergence, ordinary $I$ and absolute $|I|$, are the spaces of uniformly and absolutely convergent Fourier series:

$$
\mathscr{U}=\left\{f \in C: s_{n} f \rightarrow f I \text { uniformly }\right\} \text { and } \mathscr{A}=\left\{f \in C: s_{n} f \rightarrow f|I| \text { a.e. }\right\} .
$$

They are Banach spaces, under the norms:

$$
\|f\|_{\mathscr{U}}:=\sup _{n}\left\|s_{n} f\right\|_{C} \quad \text { and } \quad\|f\|_{\mathscr{A}}:=\sum_{k \in \mathbb{Z}}|\hat{f}(k)|=\|\hat{f}\|_{l^{1}} .
$$

It is well known that $\mathscr{A} \subset \mathscr{U} \subset C \subset L^{\infty}$ properly, where $L^{\infty}$ is the corresponding space of essentially bounded measurable functions. We shall also consider the Banach space $M$ of $2 \pi$-periodic Radon measures, under the norm $\|f\|_{M}=\sup _{n}\left\|\frac{1}{n+1} \sum_{k=0}^{n} s_{k} f\right\|_{L^{1}}$.

For the spaces $E=L^{p}(p \geq 1)$ and $L^{\infty}$, the questions of determining the largest solid space contained in $\widehat{E}$ and the smallest solid space containing $\widehat{E}$ have already been considered in [1]. Slightly expanding those results in view of the concepts of this paper, we can write the following theorem, where the standard sequence spaces are to be interpreted as the spaces of two-way sequences:

\section{Theorem 11}

[a] If $E$ is a Banach space and $L^{2} \subset E \subset L^{1}$, then $\widehat{E}_{|A K|}=\widehat{E}_{|A B|}=l^{2}$ and $l^{2}=\left\langle\widehat{L}^{2}\right\rangle \subset\langle\widehat{E}\rangle \subset\left\langle\widehat{L}^{1}\right\rangle=c_{0}$. Moreover, if $1<p \leq 2$, then $\left\langle\widehat{L}^{p}\right\rangle \subset l^{q, 2}$, where $1 / p+1 / q=1$.

[b] If $p>2$ and $1 / p+1 / q=1$, then $l^{q, 2} \subset \widehat{L}_{|A K|}^{p}$ and $\left\langle\widehat{L}^{p}\right\rangle=l^{2}$. 
[c] If $E$ is a Banach space and $\mathscr{A} \subset E \subset L^{\infty}$, then $\widehat{E}_{|A K|}=\widehat{E}_{|A B|}=l^{1}$ and $l^{1}=\langle\widehat{\mathscr{A}}\rangle \subset\langle\widehat{E}\rangle \subset\left\langle\widehat{L}^{\infty}\right\rangle \subset l^{2}$.

[d] $\widehat{M}_{|A B|}=\widehat{L}_{|A B|}^{1}=l^{2}$ and $\langle\widehat{M}\rangle=l^{\infty}$.

Proof.

[a] It was pointed out in [1] that $\widehat{L}_{|A B|}^{1}=l^{2}=\widehat{L}_{|A B|}^{2}$. Thus, $\widehat{E}_{|A B|}=l^{2}$ and, since $l^{2}$ has $A D$, by Theorem 5 we have that $\widehat{E}_{|A K|}=\widehat{E}_{|A B|}$. The corresponding statements about solid hulls were discussed in [1], and the last statement is a corollary of a result in [11].

[b] The inclusion follows from [11], and the equality $\left\langle\widehat{L}^{p}\right\rangle=l^{2}$ for $p>2$ was also discussed in [1].

[c] Since $\widehat{\mathscr{A}}=l^{1}$ is solid, $\widehat{\mathscr{A}}_{|A B|}=l^{1}$. The equality $\widehat{L}_{|A B|}^{\infty}=l^{1}$ was explained in [1]. Thus $\widehat{E}_{|A B|}=l^{1}$ and, by Theorem 5, $\widehat{E}_{|A K|}=\widehat{E}_{|A B|}$. The inclusion about solid hulls is obvious.

[d] Clearly $\widehat{L}_{|A B|}^{1} \subset \widehat{M}_{|A B|}$ and, by [a] $\widehat{L}_{|A B|}^{1}=l^{2}$. Hence $l^{2} \subset \widehat{M}_{|A B|}$. Conversely, let $\hat{f} \in \widehat{M}_{|A B|}$. Then $\|\hat{f}\|_{|\widehat{M}|}<\infty$; i.e., $\sup _{n}\left\|\frac{1}{n+1} \sum_{k=0}^{n} s_{k} f\right\|_{\left|\widehat{L}^{\prime}\right|}<$ $\infty$. Since $\widehat{L}_{|A B|}^{1}=l^{2}$, we have $\sup _{n}\left\|\frac{1}{n+1} \sum_{k=0}^{n} s_{k} f\right\|_{l^{2}}<\infty$ and therefore

$$
\frac{1}{2} \sup _{n}\left(\sum_{|k| \leq[n / 2]}|\hat{f}(k)|^{2}\right)^{1 / 2} \leq \sup _{n}\left(\sum_{|k| \leq n}\left|\left(1-\frac{k}{n+1}\right) \hat{f}(k)\right|^{2}\right)^{1 / 2}<\infty .
$$

Thus $\hat{f} \in l^{2}$. This proves the first inequality. To show that $\langle\widehat{M}\rangle=l^{\infty}$, we first note that $e \in \widehat{M}$, so that $e \cdot l^{\infty}=l^{\infty} \subset\langle\widehat{M}\rangle$. But $\widehat{M} \subset l^{\infty}$ implies $\langle\widehat{M}\rangle \subset l^{\infty}$. Thus $\langle\widehat{M}\rangle=l^{\infty}$.

Corollary. $\widehat{\mathscr{A}}_{|A B|}=\widehat{\mathscr{U}}_{|A B|}=\widehat{C}_{|A B|}=\widehat{L}_{|A B|}^{\infty}=l^{1}=\widehat{\mathscr{A}}$, and the same string of equalities is true for $|A K|$.

We consider now some newer classes of functions introduced in Fourier analysis. They are determined by other types of pointwise convergence, namely strong convergence of index $p \geq 1,[I]_{p}$, and absolute convergence of index $p \geq 1,|I|_{p}$. The latter extends the concept of absolute convergence $|I|$ in the sense that a sequence $s_{n} \rightarrow s|I|_{p}$ if and only if $s_{n} \rightarrow s I$ and $\sum k^{p-1}\left|s_{k}-s_{k-1}\right|^{p}<\infty$. The strong convergence $[I]_{p}$ lies between the absolute $|I|_{p}$ and the ordinary convergence $I$ : that is, $|I|_{p} \Rightarrow[I]_{p} \Rightarrow I$; see [14] or [16] and the references cited there. These notions were applied to trigonometric and Fourier series in a series of recent papers, [16] through [18], which led to the study of the related spaces of functions, [14], [15], [19]:

$$
\begin{aligned}
& S^{p}=\left\{f \in L^{1}: s_{n} f \rightarrow f[I]_{p} \text { a.e. }\right\}, \mathbb{S}^{p}=\left\{f \in C: s_{n} f \rightarrow f[I]_{p} \text { uniformly }\right\}, \\
& A^{p}=\left\{f \in L^{1}: s_{n} f \rightarrow f|I|_{p} \text { a.e. }\right\}, \mathscr{A}^{p}=\left\{f \in C: s_{n} f \rightarrow f|I|_{p} \text { uniformly }\right\} .
\end{aligned}
$$

For $p=1$, we write simply $S, \mathbb{S}, A$, and $\mathscr{A}$, respectively. They have many interesting properties: $\mathbb{S}^{p} \subset S^{p} \subset \bigcap_{1<r<\infty} L^{r}$ properly, but $S^{p} \not \subset L^{\infty}$; 
the classes $\mathbb{S}^{p}$ and $S^{p}$ decrease with $p$ increasing while the classes $\mathscr{A}^{p}$ are incomparable and the same is true for $A^{p} ; A=\mathscr{A} ; \mathscr{A}^{p} \subset \mathbb{S}^{p} \subset \mathscr{U}$ and $A^{p} \subset S^{p} \subset L^{p}$ properly. From the results in [14], [15], and [17] they can be described as follows.

For $p \geq 1$, let $s^{p}:=\left\{x: \frac{1}{2 n+1} \sum_{|k| \leq n}|k|^{p}\left|x_{k}\right|^{p}=o(1) \quad(n \rightarrow \infty)\right\}$. Then $\widehat{S}=\widehat{L}^{1} \cap s^{1}$ and $\widehat{S} \subset s^{1}$ properly, $\widehat{S}^{p}=s^{p}=\left\{x: n^{p-1} \sum_{|k| \geq n}\left|x_{k}\right|^{p}=o(1)\right.$ $(n \rightarrow \infty)\}$ for $p>1$, and $\widehat{\mathbb{S}}^{p}=\widehat{C} \cap \widehat{S}^{p}$ for $p \geq 1$. For $p \geq 1, \widehat{A}^{p}=a^{p}:=$ $\left\{x: \sum_{k \in \mathbb{Z}}|k|^{p-1}\left|x_{k}\right|^{p}<\infty\right\}$ and $\widehat{\mathscr{A}}^{p}=\widehat{C} \cap \widehat{A}^{p}$. They are Banach spaces under the corresponding norms:

$$
\begin{aligned}
& \|f\|_{S}=\|f\|_{L^{1}}+\|f\|_{[1]} ;\|f\|_{S^{p}}=\|f\|_{[p]} \text { for } p>1 ; \\
& \|f\|_{S^{p}}=\|f\|_{\mathscr{C}}+\|f\|_{[p]} \text { for } p \geq 1 ; \\
& \|f\|_{A^{p}}=\|f\|_{|p|} \text { and }\|f\|_{\mathscr{A}^{p}}=\|f\|_{\mathscr{U}}+\|f\|_{|p|} \text { for } p \geq 1,
\end{aligned}
$$

where

$$
\begin{gathered}
\|f\|_{[p]}=\sup _{n}\left(\frac{1}{2 n+1} \sum_{|k| \leq n}(|k|+1)^{p}|\hat{f}(k)|^{p}\right)^{1 / p} \text { and } \\
\|f\|_{|p|}=\left(|\hat{f}(0)|^{p}+\sum_{k \in \mathbb{Z}, k \neq 0}|k|^{p-1}|\hat{f}(k)|^{p}\right)^{1 / p} .
\end{gathered}
$$

\section{Theorem 12}

[a] $\widehat{S}_{|A K|}^{p}=\widehat{S}_{|A B|}^{p}=s^{p}=\widehat{S}^{p}=\left\langle\widehat{S}^{p}\right\rangle$ for $p>1$.

[b] $\widehat{S}_{|A K|}=\widehat{S}_{|A B|}=l^{2} \cap s^{1} \quad\left(l^{2}\right.$ and $\mathbb{S}^{1}$ are incomparable $)$.

[c] $\widehat{\mathbb{S}}_{|A K|}^{p}=\widehat{\mathbb{S}}_{|A B|}^{p}=l^{1} \cap s^{p} \quad\left(l^{1} \not \subset s^{p}\right.$ except for $p=1$ and $\left.s^{p} \not \subset l^{1}\right)$.

[d] $\left\langle\widehat{\mathbb{S}}^{p}\right\rangle=s^{p}=\widehat{S}^{p}$ for $p>1$ and $\langle\widehat{\mathbb{S}}\rangle \subset l^{2} \cap s^{1}=\widehat{S}_{|A B|}$.

Proof

[a] By the above remarks, $\widehat{S}^{p}=s^{p}$ for $p>1$. Since $s^{p}$ is solid and has the property $A D$, the statement follows from Theorem 5 .

[b] $\widehat{S}=\widehat{L}^{1} \cap s^{1}$ and, consequently, $\widehat{S}_{|A B|} \subset \widehat{L}_{|A B|}^{1} \cap s^{1}$, since $s^{1}$ is solid. By Theorem 11 [a], $\widehat{L}_{|A B|}^{1}=l^{2}$, and therefore $\widehat{S}_{|A B|} \subset l^{2} \cap s^{1}$. Conversely, $l^{2} \cap s^{1} \subset \widehat{L}_{|A B|}^{1} \cap s^{1} \subset \widehat{S}_{|A B|}$. Moreover, by Theorem 5, $\widehat{S}_{|A K|}=\widehat{S}_{|A B|}$.

[c] By the above remarks, $\mathbb{S}^{p}=C \cap S^{p}$ and, by the corollary of Theorem $11, \widehat{C}_{|A B|}=l^{1}$. Hence, by statement $[a], \widehat{\mathbb{S}}_{|A B|}^{p} \subset l^{1} \cap s^{p}$ and conversely $l^{1} \cap s^{p} \subset \widehat{C}_{|A B|} \cap \widehat{S}_{|A B|}^{p} \subset \widehat{\mathbb{S}}_{|A B|}^{p}$. The equality $\widehat{\mathbb{S}}_{|A K|}^{p}=\widehat{\mathbb{S}}_{|A B|}^{p}$ is clear from Theorem 5.

[d] Since $\mathbb{S}^{p}=C \cap S^{p}$, clearly $\left\langle\widehat{\mathbb{S}}^{p}\right\rangle \subset\left\langle\widehat{S}^{p}\right\rangle=s^{p}$ for all $p \geq 1$. To show the converse inclusion for $p>1$, we refer to a result due to Salem [2, vol. 1, p. 
335], noting first that $x \in s^{p}$ implies that

$$
\sum_{k=2}^{\infty} \frac{1}{k \sqrt{\log k}}\left(\sum_{|i| \geq k}\left|x_{i}\right|^{2}\right)^{1 / 2}<\infty
$$

Since $s^{p} \subset s^{2}$ for $p>2$, it clearly suffices to assume that $1<p \leq 2$. Taking $1<p \leq 2$ for $x \in s^{p}$ we have,

$$
\left(\sum_{|i| \geq k}\left|x_{i}\right|^{2}\right)^{1 / 2} \leq\left(\sum_{|i| \geq k}\left|x_{i}\right|^{p}\right)^{1 / p}=O\left(\frac{1}{k^{1 / q}}\right), \text { where } 1 / p+1 / q=1,
$$

from which it follows that $(*)$ is satisfied. We now show that $s^{p} \subset l^{\infty} \cdot \hat{S}^{p}$. For $x \in s^{p}$, let $x=x^{r}+x^{l}$, where $x_{k}^{r}=x_{k}$ for $k \geq 0, x_{k}^{r}=0$ for $k<0$, and $x_{k}^{l}=0$ for $k \geq 0, x_{k}^{l}=x_{k}$ for $k<0$. By the above argument, both $x^{r}$ and $x^{l}$ satisfy $(*)$. Consequently, by Salem's theorem, there exists a sequence $\left(\alpha_{k}^{r}\right)_{k=0}^{\infty}$ such that the series

$$
\sum_{k=0}^{\infty} x_{k}^{r} \cos \left(k t-\alpha_{k}^{r}\right)
$$

converges uniformly and is therefore the Fourier series of its sum function $g \in C$. Hence $\hat{g}_{c}(k)=x_{k}^{r} \cos \alpha_{k}^{r}$ and $\hat{g}_{s}(k)=x_{k}^{r} \sin \alpha_{k}^{r}$. Expressed in complex form, $g$ is the uniform sum of the series

$$
\sum_{k \in \mathbb{Z}} \hat{g}(k) e^{i k t}, \text { where } \hat{g}(k)=\frac{1}{2} x_{k}^{r} e^{-i \alpha_{k}^{r}}, \hat{g}(-k)=\frac{1}{2} x_{k}^{r} e^{i \alpha_{k}^{r}} \text {. }
$$

Consequently, defining a two-way sequence $y$ by $y_{k}=2 e^{i \alpha_{k}^{r}}$ for $k \geq 0$ and $y_{k}=0$ for $k<0$, we have $x^{r}=y \cdot \hat{g}$ where $y \in l^{\infty}$. But clearly $\hat{g} \in s^{p}=\hat{S}^{p}$, and therefore $x^{r}=y \cdot \hat{g} \in l^{\infty} \cdot S^{p}$. In the same way we can show that $x^{l} \in l^{\infty} \cdot \hat{S}^{p}$. Consequently $x \in l^{\infty} \hat{\otimes} \hat{S}^{p} \subset\left\langle\widehat{S}^{p}\right\rangle$.

The corresponding properties for the spaces $\widehat{A}^{p}$ and $\widehat{\mathscr{A}}^{p}$ are proved similarly, noting that $a^{p} \subset s^{p}$ so that $x \in a^{p}$ implies $(*)$.

\section{Theorem 13}

[a] $\widehat{A}_{|A K|}^{p}=\widehat{A}_{|A B|}^{p}=a^{p}=\widehat{A}^{p}=\left\langle\widehat{A}^{p}\right\rangle$ for $p \geq 1$.

[b] $\widehat{\mathscr{A}}_{|A K|}^{p}=\widehat{\mathscr{A}}_{|A B|}^{p}=l^{1} \cap \mid a^{p}$ for $p \geq 1$.

[c] $\left\langle\widehat{\mathscr{A}}^{p}\right\rangle=a^{p}=\widehat{A}^{p}$ for $p>1$ and $\left\langle\widehat{\mathscr{A}\rangle}=l^{1}\right.$.

\section{REFERENCES}

1. J. M. Anderson and A. L. Shields, Coefficient multipliers of Bloch functions, Trans. Amer. Math. Soc. 224 (1976), 255-265.

2. N. Bary, A treatise on trigonometric series, vols. 1 and 2, Pergamon Press, New York, 1964.

3. G. Bennett and N. J. Kalton, Inclusion theorems for $K$-spaces, Canad. J. Math. 25 (1973), 511-524. 
4. M. Buntinas, Convergent and bounded Cesàro sections in FK-spaces, Math. Z. 121 (i971), 191-200.

5. __ On Toeplitz sections in sequence spaces, Math. Proc. Cambridge Philos. Soc. 78 (1975), 451-460.

6. __ Products of sequence spaces, Analysis 7 (1987), 293-304.

7. M. Buntinas and G. Goes, Products of sequence spaces and multipliers, Radovi Mat. 3 (1987), 287-300.

8. D. J. H. Garling, The $\beta$ - and $\gamma$-duality of sequence spaces, Proc. Cambridge Philos. Soc. 63 (1967), 963-981.

9. __ On topological sequence spaces, Proc. Cambridge Philos. Soc. 63 (1967), 997-1019.

10. C. Goffman and G. Pedrick, A first course in functional analysis, Prentice-Hall, Englewood Cliffs, NJ, 1965.

11. C. N. Kellogg, An extension of the Hausdorff-Young Theorem, Michigan Math. J. 18 (1971), 121-127.

12. J. J. Sember, On unconditional section boundedness in sequence spaces, Rocky Mountain J. Math. 7 (1977), 699-706.

13. J. Sember and M. Raphael, The unrestricted section properties of sequences, Canad. J. Math. 31 (1979), 331-336.

14. I. Szalay and N. Tanović-Miller, On Banach spaces of absolutely and strongly convergent Fourier series, Acta Math. Hung. (1989), (to appear).

15. __ On Banach spaces of absolutely and strongly convergent Fourier series, II, Acta Math. Hung. (to appear).

16. N. Tanović-Miller, On strong convergence of trigonometric and Fourier series, Acta Math. Hung. 42 (1983), 35-43.

17. __ On a paper of Bojanić and Stanojević, Rendiconti Cir. Mat. Palermo 34 (1985), 310324.

18. _ Strongly convergent trigonometric series as Fourier series, Acta Math. Hung. 47, (1986), 127-135.

19. __, On Banach spaces of strongly convergent trigonometric series, J. Math. Anal. and Appl. (to appear).

20. K. Zeller, Allgemeine Eigenschaften von Limitierungsverfahren, Math. Z. 53 (1951), 463487.

Department of Mathematical Sciences, loyola University of Chicago, Chicago, IlliNOIS 60626

Department of Mathematics, University of Sarajevo, 71000 Sarajevo, Yugoslavia 